\title{
Semblanza del Dr. Arnaldo Tejeiro Fernández*
}

\section{Norma Eneida Ríos Massabot}

Doctora en Ciencias Médicas. Sociedad Cubana de Salud Pública. La Habana, Cuba.

\section{NTRODUCCI ÓN}

Conocí al Doctor Arnaldo Fermín Tejeiro Fernández en 1970 cuando se incorporó a trabajar en la Dirección de Estadística del Ministerio de Salud Pública. El entonces Director, Dr. Francisco Rojas Ochoa, promovía en Cuba el desarrollo de las estadísticas del país no solo como el registro de datos continuos con calidad, sino también como herramienta indispensable para el desarrollo de investigaciones con el rigor científico requerido. Rojas sabia de los conocimientos de Tejeiro en Epidemiología, Estadísticas y Metodología de la Investigación, pues trabajaron juntos en la provincia de Camagüey, el primero como Director Provincial y el segundo como Vicedirector de Higiene y Epidemiología.

Desde entonces los tres compartimos las actividades docentes, investigativas y de servicio de la dirección haciendo énfasis cada uno en su responsabilidad. A ambos le debo haber completado mi formación en Salud Publica.

El haber compartido con el doctor Tejeiro el trabajo diario por más de 20 años, formar parte de la representación de Cuba en reuniones internacionales, realizar investigaciones, aun después de estar ambos jubilados, así como precisar aspectos de su vida, que conocí por sus referencias en la conferencia del profesor Gregorio Delgado García titulada: "Dr. Arnaldo F. Tejeiro Fernández, Maestro de Epidemiólogos Cubanos", me permiten comentarles aspectos de su vida y de su labor en la salud pública cubana.

\section{NACI MI ENTO, ESTUDI OS Y EJ ERCI CI O PROFESI ONAL}

Nació en el poblado de Madruga, provincia La Habana, el 7 de octubre de 1923. Su infancia trascurrió en el central "Merceditas", hoy "Gregorio A. Mañalich". La enseñanza primaria la realizó en la escuela pública del central y sus estudios secundarios en el instituto de segunda enseñanza de Güines. Ya desde épocas tempranas, por necesidades económicas trabajaba como profesor repasando alumnos para ingresar al bachillerato y como repasador de distintas asignaturas a alumnos de años anteriores al que él cursaba. Terminado el bachillerato comienza 
sus estudios en la carrera de medicina en el curso escolar 1941-1942, uno de los más numerosos para aquella época, pues incluía a los graduados de bachiller del año anterior que no pudieron comenzar la carrera por estar cerrada la escuela.

Ya de estudiante universitario comienza a trabajar como revisor de la mecanografía en esténcil de las conferencias que para los alumnos de medicina confeccionaba el "repasador" (J osé I sidro Hernández López). De esta forma adquiría las conferencias exentas de pago y algún salario para mejorar su economía. A partir del cuarto año de la carrera comienza a trabajar en Casas de Socorro y después en el dispensario de Profilaxis de Enfermedades Venéreas de La Habana. Durante su trabajo en este dispensario pudo observar la precaria situación económica y los problemas sociales que vivían los pacientes que acudían a solicitar atención médica. En el último año de la carrera logró por concurso una plaza de alumno externo del Hospital Universitario.

Concluido los 7 años de estudio de la carrera comenzó su tesis de grado titulada: "Estudio Estadístico de la Presión Arterial en Cuba". Para ello visitó varios lugares del país, entre ellos la Ciudad de Camagüey, donde conoció a Migdalia Noy Peláez, con quien contrajo matrimonio después de graduado y compartió su vida.

Los ejercicios para obtener el grado de Doctor en Medicina los realizó en el Hospital Universitario, en los que obtuvo calificación de sobresaliente con recomendación de que la tesis fuera publicada. El 26 de noviembre de 1948 obtuvo el título de Doctor en Medicina.

Con los conocimientos teóricos adquiridos en la carrera, las prácticas en las Casas de Socorro y el Hospital Universitario, comenzó a ejercer su carrera en el poblado de Santa Cruz de los Pinos, del municipio San Cristóbal, provincia de Pinar del Río. En el pueblo era el único médico, el anterior falleció antes de su llegada. Conocí que para algunos habitantes del lugar él fue más que un amigo, lo consideraban un familiar en quien confiar. Durante los once años que vivió en este pueblo, para sobrevivir económicamente, tuvo que trabajar como químico en la Industria Lechera San Bernardo S.A., además en los últimos años también tuvo una consulta de cardiología en la ciudad de Pinar del Río.

En el año 1959 sus compañeros de carrera: Ramón Martínez Rodríguez y Carmen Pascual J acobino, ambos integrantes de la Columna Sanitaria, recorrían la provincia de Pinar para determinar la situación sanitaria y los recursos con que contaba esta territorio, se encontraron con Tejeiro aún de médico en Santa Cruz de Los Pinos. Fueron ellos los que le informaron a Tejeiro acerca de la necesidad que tenía el país de formar epidemiólogos y de ellos especialistas en malarialogía. El aspiró y fue seleccionado para ir a formarse como malariólogo en la escuela de la República de Venezuela. Para allí partió acompañado de su esposa y sus dos hijos. La beca incluía además, para completar su formación en esta especialidad, un curso de un mes de duración sobre "Organización de la Erradicación de Paludismo"en México, D.F. y otro sobre la resistencia de los mosquitos a los insecticidas en la Comisión de Erradicación de Paludismo en El Salvador. En este posgrado obtuvo calificación de 100 puntos en todas las asignaturas y en el examen final. En la escuela se exhibe un cuadro con sus notas que dice: "Podrá pasar por la escuela otro alumno igual que él, pero no mejor". Fue la calificación más alta desde la fundación de la escuela en 1943.

A su regreso a Cuba fue nombrado responsable de la erradicación del paludismo en la región II del país, que según la división política anterior estaba constituida por la provincia de Camagüey y la parte este de Las Villas; conjuntamente con este cargo 
fue nombrado Subdirector Provincial de Higiene y Epidemiología de la provincia de Camagüey. Durante todo este tiempo mantuvo una participación activa en las tareas revolucionarias. Recuerdo sus referencias sobre el ciclón Flora en 1963.

En enero de 1964 fue trasladado a Santiago de Cuba, donde ocupó el cargo de Subdirector de Higiene y Epidemiología de la región Oriente Sur y el de Director Nacional de Erradicación del Paludismo.

Su estancia en Santiago coincide con los primeros años de la escuela de medicina en la Universidad de Oriente, de la que fue Director. Tuvo participación activa en el desarrollo docente de este centro y de conjunto con los alumnos participó en los trabajos de instalación de locales apropiados para impartir clases de algunas asignaturas y en las zafras del pueblo.

Sus habilidades en la estadística las había explotado primero al trabajar como enumerador en el censo de 1943, siendo todavía alumno de medicina, trabajo del que me hablaba con orgullo, ya que por motivos laborales tuvimos que consultar la publicación del informe final de este censo en varias oportunidades, posteriormente en su tesis de grado, para la que realizó un trabajo estadístico y por último, en su formación como epidemiólogo.

Como J efe del Departamento de Medicina Preventiva en la Universidad, escribió varios textos de epidemiología, estadística y metodología de la investigación. Compartió la asignatura de Estadística con el Doctor Orlando Aguirre y los Técnicos Hidelgardis Corrales Rosales, Manuel Dócil López y Eunomia Marañón Pérez. Posteriormente se incorporó al equipo el Dr. Rigoberto Yoanis Verdecía, Jefe Provincial de Estadística y Especialista en Epidemiología.

En 1968 asistió a un seminario internacional realizado en Francia, el cual tuvo como objetivo demostrar las posibilidades de la informática médica en el campo de la medicina, a este seminario también asistieron los Doctores Orlando Aguirre de la escuela de medicina de Santiago de Cuba, José Luis Álvaro-Díaz Artiles de la escuela de medicina de La Habana y Guillermo Halley jefe del Registro Nacional del Cáncer.

Erradicado el paludismo en el país, con el ultimo caso en 1967, y encontrándose enfermo su padre en la provincia de La Habana, se traslada para esta ciudad a principios de 1970, donde fue nombrado J efe del Grupo de Investigaciones en la Dirección Nacional de Estadística.

En este grupo, a principios de 1970, Tejeiro contaba con la participación de Cándido López Pardo. Ellos dos y Rojas impartían clases en los cursos de estadística y metodología de la investigación a los especialistas de los institutos. La cantidad de integrantes del grupo fue creciendo, a ellos se les incorporarían la trabajadora de la dirección y estudiante de matemáticas Luisa Álvarez Vázquez y los alumnos de la recién iniciada Residencia en Bioestadística, María Amparo Pascual, Rene Suárez Martínez, Eddy Gómez Cabalé así como los de los cursos siguientes y los alumnos de los dos últimos años de la Licenciatura en Matemáticas de la Universidad de La Habana. Un grupo importante de estos alumnos después de graduados, se incorporaron a trabajar en instituciones de salud pública. Recuerdo a Mercedes Rubén Quesada, Carlos Sánchez Texido, Jorge Bacallao Gallestey, Luís Carlos Silva y Antonio Rubí y al grupo también se le unieron psicólogos y sociólogos. Todos bajo las orientaciones directas de Tejeiro formaron un grupo multidisciplinario que logró su máximo desarrollo después de su traslado al Instituto de Desarrollo de la Salud. En este centro Tejeiro ocupó el cargo de Asesor de la Dirección hasta 1980. 
Mencionar las investigaciones que contaron con la asesoría de Tejeiro sería una lista muy extensa, las más relevantes en la década de los años setenta que contaron con su asesoramiento y el de su equipo, respondían a los diferentes programas que desarrollaba la Salud Pública Cubana. Entre ellas están:

- Crecimiento y Desarrollo del Niño y el Adolescente Cubano. Este estudio surge como consecuencia del análisis y reestructuración del Programa Materno Infantil en 1970. En pediatría se utilizaban tablas de peso y talla de países con superior grado de desarrollo socioeconómico que el nuestro y con características étnicas y antropométricas diferentes, por lo que se decide realizar este trabajo entre 1972 y 1974, bajo la dirección del destacado pediatra Dr. José Jordán Rodríguez con asesoramiento de destacados especialistas británicos en pediatría y de estadística.

- Mortalidad Perinatal en Cuba. Desarrollado bajo la dirección del profesor J osé A. Gutiérrez Muñiz y del profesor Francisco Rojas Ochoa. Esta investigación aportó valiosa información para el análisis de múltiples factores causales de la mortalidad de este período de la vida, contó con la participación de obstetras, neonatólogos, patólogos, matemáticos, técnicos de estadística y consultoría a especialistas británicos.

- Efectos Biológicos y Sociales en la Mortalidad Perinatal. Cuba fue invitada por la Organización Mundial de la Salud a participar en una investigación sobre mortalidad perinatal en unión de ocho países desarrollados. Tejeiro representó a nuestro país en todas las reuniones, y en la redacción del informe final.

También contaron con su asesoría las investigaciones sobre prevalencia y características de la morbilidad. Entre ellas:

- Prevalencia de la Diabetes Mellitus en el Regional Plaza de Revolución, de los Drs. J. A. Muñoz y Oscar Mateo de Acosta.

- Prevalencia del Asma Bronquial en San Antonio de los Baños, del Profesor Adolfo Rodríguez de la Vega.

- Prevalencia de la Epilepsia, de la Dra. Maria Amparo Pascual López.

- Estudio de la Morbilidad Percibida, del Dr. Eddy Gómez Cavalé.

- Prevalencia del Asma en Cuba, del Profesor Adolfo Rodríguez de la Vega.

Durante la década del 70 impartió clases de estadística descriptiva a residentes de epidemiología, bioestadística y administración de salud. De esta época son sus textos: "El Examen Masivo de la Población como Instrumento de Salud Pública" y "La Serie Cronológica", este último de obligada consulta aún en nuestros días.

En 1980 comienza a trabajar nuevamente en la Dirección de Estadística, en esta oportunidad como Asesor de la Dirección. Tejeiro tuvo participación activa en 3 temas de investigación del Consejo de Ayuda Mutua Económica (CAME). Estos fueron:

- Diccionario Terminológico Multilingüe en la Esfera de la Higiene Social, dirigido por la autora de esta entrega. Participó en una de las reuniones internacionales. El informe final fue publicado por el CAME en idioma ruso y el Ministerio de Salud Publica publicó en español la parte cubana con el titulo "Definiciones de los Principales Términos y Conceptos sobre la Morbilidad de la Población."

- Causas Múltiples de Morbilidad Perinatal, dirigido por el pediatra Héctor Duyos Gato. En la publicación se explica con ejemplos cómo se anotan correctamente las causas de muerte en el certificado de defunción perinatal. 
- Metódica para el estudio de la Morbilidad en Consulta, dirigido por la Dra. Carmen Luisa Casanovas Casas.

Durante el primer quinquenio de la década de los años 80 compartimos con Tejeiro la dirección y ejecución de un proyecto de investigación auspiciada por la Organización Panamericana de la Salud (OPS), que tenía como objetivo que los países profundizaran en el análisis de la mortalidad. Una vez terminada la investigación, el empeño y entusiasmo de Tejeiro logró interesarme en completar el estudio con el análisis de los indicadores del estado de salud de la población. Este estudio se titula "Evolución de la mortalidad en Cuba, analizando un Trienio de cada Década del Periodo Revolucionario."

A finales del quinquenio 1986-1990, y también auspiciada por la OPS, se realizó la investigación titulada "Calidad de la Certificación Médica de Defunción". Se revisaron los certificados médicos de una muestra representativa de los fallecidos en el país durante 4 meses de 1988. Las anotaciones realizadas en los certificados fueron evaluadas con la documentación médica existente en las unidades asistenciales en que fueron atendidos y la no médica con el registro de dirección del área de residencia.

Durante este decenio impartió clases de estadística de morbilidad a epidemiólogos, de la República de Nicaragua como profesor contratado por la Organización Panamericana de la Salud.

En varias oportunidades fue miembro de la delegación cubana que acompañó al Ministro de Salud Pública, Doctor Sergio del Valle Jiménez a la Asamblea Mundial de la Salud. Además fue miembro de la delegación cubana a la Conferencia Internacional para la Décima Revisión de la Clasificación Internacional de Enfermedades.

Después de su jubilación trabajamos juntos en la investigación "Causas Múltiples de Muerte" y en el informe a la Organización Panamericana de la Salud sobre la evaluación de la parte cubana del Plan Regional de Acción para la Reducción de la Mortalidad Materna en Las Américas en la etapa 1988-93.

En los años de trabajo compartidos con Tejeiro conocí de su modestia, sencillez, su aporte a la Salud Pública Cubana, de su contribución al desarrollo de la actividad científica del Ministerio de Salud Pública y de los reconocimientos recibidos por su extensa labor: Especialista de II Grado en Epidemiología y Administración, Profesor e Investigador Titular, Doctor en Ciencia Médicas, Miembro de Honor de la Sociedad Cubana de Higiene y Epidemiología y de la Sociedad Cubana de Salud Pública. En 1983 Premio de la OPS en Administración de Salud. Fue para la mayoría de los aquí presentes profesor y amigo, siempre dispuesto a brindar la orientación correcta.

Muchas gracias

Recibido: 27 de abril de 2009.

Aprobado: 23 de junio de 2009. 
Norma Eneida Ríos Massabot. Sociedad Cubana de Salud Pública. La Habana, Cuba.

* Presentado en el Simposio "Arnaldo Tejeiro Fernández", Investigación Cualitativa en Salud Pública, 23 de junio de 2009. 